\title{
The Distribution of Dengue Virus Serotype in Quang Nam Province (Vietnam) during the Outbreak in 2018
}

\author{
Duong Q. Phan ${ }^{1}$, Linh D. N. Nguyen ${ }^{2}$, Son T. Pham ${ }^{3}{ }^{\circledR}$, Tai Nguyen ${ }^{1}$, Phuong T. T. Pham ${ }^{2}$, Suong T. H. Nguyen ${ }^{2}$, \\ Dien T. Pham ${ }^{2}$, Huong T. Pham ${ }^{4}$, Duy K. Tran ${ }^{5}$, Sa H. Le ${ }^{5}$, Tung T. Pham ${ }^{2}$, Kieu C. D. Nguyen ${ }^{6}$, \\ Gianna Dipalma ${ }^{6}\left(\mathbb{D}\right.$, Alessio Danilo Inchingolo ${ }^{6} \mathbb{D}$, Prisco Piscitelli ${ }^{7,8, *} \mathbb{D}$, Alessandro Miani ${ }^{8,9}$, \\ Scacco Salvatore ${ }^{10}\left(\mathbb{D}\right.$, Stefania Cantore ${ }^{6}(\mathbb{D})$, Sergey K. Aityan ${ }^{11}$, Andrea Ballini ${ }^{12,13, *(D)}$, \\ Francesco Inchingolo ${ }^{6, *}+\mathbb{D}$, Ciro Gargiulo Isacco ${ }^{6,+}$ and Van H. Pham ${ }^{2,+}$
}

\section{check for} updates

Citation: Phan, D.Q.; Nguyen, L.D.N.; Pham, S.T.; Nguyen, T.; Pham, P.T.T.; Nguyen, S.T.H.; Pham, D.T.; Pham, H.T.; Tran, D.K.; Le, S.H.; et al. The Distribution of Dengue Virus Serotype in Quang Nam Province (Vietnam) during the Outbreak in 2018. Int. J. Environ. Res. Public Health 2022, 19, 1285. https://doi.org/ 10.3390/ijerph19031285

Academic Editor: Richard Paul

Received: 20 November 2021

Accepted: 20 January 2022

Published: 24 January 2022

Publisher's Note: MDPI stays neutral with regard to jurisdictional claims in published maps and institutional affiliations.

Copyright: (C) 2022 by the authors. Licensee MDPI, Basel, Switzerland. This article is an open access article distributed under the terms and conditions of the Creative Commons Attribution (CC BY) license (https:// creativecommons.org/licenses/by/ $4.0 /)$.
1 The General Hospital of Quang Nam, Xã Tam Hiệp 51000, Vietnam; bsphanquangduong@gmail.com (D.Q.P.); tainguyen.bvtwqn@gmail.com (T.N.)

2 Faculty of Medicine, Phan Chau Trinh University, Danang City 550000, Vietnam; linh.nguyendo@pctu.edu.vn (L.D.N.N.); phuong.ptt@pctu.edu.vn (P.T.T.P.); suong.nth@pctu.edu.vn (S.T.H.N.); dien.pt@pctu.edu.vn (D.T.P.); tungyduoc1218@gmail.com (T.T.P.) van.pham@pctu.edu.vn (V.H.P.)

3 School of Medicine, The University of Sydney, Sydney, NSW 2006, Australia; truongson.pham@gmail.com

4 Pham Ngoc Thach University of Medicine, Ho Chi Minh City 70000, Vietnam; phamthienhuong@gmail.com

5 NK-Biotek Laboratories, Ho Chi Minh City 70000, Vietnam; khanhduytransh@gmail.com (D.K.T.); sa.shane.le@outlook.com (S.H.L.)

6 Department of Interdisciplinary Medicine, University of Bari “Aldo Moro", 70124 Bari, Italy; drkieukaren@gmail.com (K.C.D.N.); giannadipalma@tiscali.it (G.D.); ad.inchingolo@libero.it (A.D.I.); stefaniacantore@pec.omceo.bari.it (S.C.); drciroisacco@gmail.com (C.G.I.)

7 Euro Mediterranean Scientific Biomedical Institute (ISBEM), 72023 Mesagne, Italy

8 Italian Society of Environmental Medicine (SIMA), 20123 Milan, Italy; alessandro.miani@gmail.com

9 Department of Environmental Science and Policy, University of Milan, 20132 Milan, Italy

10 Department of Basic Medical Sciences, Neurosciences and Sense Organs, University of Bari "Aldo Moro", 70124 Bari, Italy; salvatore.scacco@uniba.it

11 Department of Multidisciplinary Research Centre, Lincoln University, Oakland, CA 94612, USA; aityan@lincolnuca.edu

12 School of Medicine, University of Bari "Aldo Moro", 70124 Bari, Italy

13 Department of Precision Medicine, University of Campania “Luigi Vanvitelli", 80138 Naples, Italy

* Correspondence: piscitelli@isbem.it (P.P.); andrea.ballini@me.com (A.B.); francesco.inchingolo@uniba.it (F.I.); Tel.: +39-083-171-3512 (P.P.)

$\dagger$ These authors contributed equally to this work.

\begin{abstract}
Objectives: Quang Nam province in the Centre of Vietnam has faced an outbreak of dengue hemorrhagic fever (DHF) in 2018. Although DHF is a recurrent disease in this area, no epidemiological and microbiological reports on dengue virus serotypes have been conducted mainly due to lack of facilities for such a kind of advanced surveillance. The aim of this study was to detect different dengue virus serotypes in patients' blood samples. Design and Methods: Suspected cases living in Quang Nam province (Vietnam) and presenting clinical and hematological signs of dengue hemorrhagic fever were included in the study. The screening was performed, and the results were compared by using two methodologies: RT real-time PCR (RT-rPCR) and the Dengue NS1 rapid test. Results: From December 2018 to February 2019, looking both at RT-rPCR [+] and NS1 [+] methodologies, a total of 488 patients were screened and 336 were positive for dengue virus detection (74 children and 262 adults); 273 of these patients (81.3\%) underwent viral serotype identification as follows: $12.82 \%$ (35/273) D1 serotype, 17.95\% (49/273) D2, 0.37\% (1/273) D3, $68.50(187 / 283)$ D4, and 0.37\% (1/273) D2+D4 serotypes. The RT-rPCR outcomes showed higher sensitivity during the first three days of infection compared to NS1 $(92.3 \%$ vs. $89.7 \%)$. The NS1 increased sensitivity after the first 3 days whilst the RT-rPCR decreased. Conclusions: Advanced surveillance with dengue virus serotypes identification, if performed routinely, may help to predict and prevent further DHF epidemics based on the exposure of the different serotypes during different periods that lead to the intensification of disease severity as a consequence of antibody-dependent enhancement (ADE).
\end{abstract}


Keywords: dengue hemorrhagic fever; dengue virus serotypes; Quang Nam; Vietnam

\section{Introduction}

Dengue virus (DENV) is the main cause of dengue hemorrhagic fever (DHF), and it is transmitted by the Aedes aegypti (A. aegypti) mosquito, a typical breed of tropical and subtropical areas. Clinically, DHF is classified as (i) silent DHF without warning signs, (ii) DHF with warning signs (mucosal bleeding, lethargy, persistent vomiting, fluid accumulation, increasing hematocrit, and decreasing platelets, etc.), and (iii) severe DHF, which is characterized by severe plasma leakage, severe hemorrhagic events, and organ failure. The disease can progress from mild to severe and death [1,2]. According to the World Health Organization (WHO), 50-100 million DHF occur annually, corresponding to a 30-fold increase compared to five decades ago [3,4]. In 2009, the number of infections dramatically increased with the DHF outbreaks in many countries in tropical and subtropical regions, including North and South America, Southern Europe, and especially in Southeast Asian Countries such as Vietnam [3,5]. In Vietnam, four DENV serotypes were detected linked to different outbreaks every year [6]. As reported by NhaTrang Pasteur Institute in 2018, about 17,200 DHF cases were diagnosed in 11 cities from Quang Binh to Binh Thuan and Quang Nam provinces. In the first 6 months of 2019, a total of 96,000 cases were reported in Vietnam, showing a three-fold increase compared to the previous year, with a mortality rate of $0.007 \%$ [7]. According to the report of Quang Nam Center for Disease Control, during the first 7 months of 2019, the number of DHF increased by three-fold in comparison to 2018 [8]. The DENV was classified into four serotypes: DENV-1, DENV-2, DENV-3, and DENV-4 [1,3]. The different serotypes could influence manifestation as well as the severity of DHF [9], resulting in increased risk if patients are re-infected with DENV-2 or DENV-3 compared to DENV-4 [10]. Data from Cuba, Hawaii, and Thailand revealed a similar figure: patients affected by DENV-1 showed a higher risk of being affected by severe DHF in case of secondary infections with DENV-2 or DENV-3 serotypes [11]. A different study on DHF conducted in Vietnam demonstrated that the clinical patterns of re-infected patients worsen when DENV-2 is followed by DENV-1 re-infection; then if DEN V-4 is followed by DENV-1; when DENV-3 is followed by DENV-2, and finally if DENV-3 is followed by DENV-4 [12].

The antibody-dependent enhancement (ADE) was applied to explain why under 1-year-old infants infected with a DENV serotype different from that of the mother showed a higher risk of developing severe DHF [13]. These data suggest that the detection of prevalent DENV serotypes is very important to help clinicians in treating the disease, as well as to assess more specific measures according to an accurate epidemiological assessment.

\section{Materials and Methods}

This was a cross-sectional study. Patients enrolled in the study were all subjects clinically diagnosed with DHF and hospitalized at Quang Nam General Hospital, in accordance with decision number 1499 issued on May 17th, 2011, by the Ministry of Health. Patients enrolled were living in or coming from an area with an outbreak or endemic of DHF within 14 days with sudden onset of high fever for 2-7 days and at least 2 of the following signs: (1) hemorrhagic signs at different levels: positive tourniquet test, cutaneous petechiae/purpura, gum or nose bleeding; (2) headache, loss of appetite, and nausea; (3) skin congestion, rash, or both; (4) muscular pain, joint pain, and orbital pain; (5) irritability and lethargy; (6) abdominal pain localized on the hypochondriac region. Each enrolled patient was given informed consent to sign in accordance with the revisited 2013 Helsinki Declaration. Blood samples were collected with 2-3 mL using EDTA-containing tubes.

Each sample was given a status including age, date of birth, gender, and the starting day of fever, together with the details regarding epidemic information. After collection, the blood samples were stored in the refrigerator $\left(2-8{ }^{\circ} \mathrm{C}\right)$ for up to $8 \mathrm{~h}$ and sent to the Research Center of Phan Chau Trinh University in the foam box with gel ice packs. At 
the Research Center, the blood samples were immediately centrifuged at 3000 RPM for $10 \mathrm{~min}$ to isolate blood plasma and immediately analyzed by one-step reverse transcriptase real-time PCR (RT-rPCR) for DENV detection and for viral serotype identification that was referred from Gilberto A. Santiago (CDC) et al. [14,15]. Briefly, nucleic acids were extracted completely from $200 \mu \mathrm{L}$ plasma on the KingFisher FLEX system (Thermo, Waltham, MA, USA) using ${ }^{N K}$ DNARNAprep-MAGBEAD extraction kits manufactured by Nam Khoa company [16,17]. Extracted fluid $(5 \mu \mathrm{L})$ was added into PCR 0.1 tubes of Rotor Gen Q MDx 5plex Platform (Qiagen, Kuala Lumpur, Malaysia), containing $20 \mu \mathrm{L}$ one step RT rPCR mix made from Thermo AgPath-ID ${ }^{\text {TM }}$ One-Step RT-PCR (Thermo, USA) with four specific primer pairs and four specific probes for four different DENV serotypes, that were: D1-F (CAA AAG GAA GTC GTG CAA TA), D1-R (CTG AGT GAA TTC TCT CTA CTG AAC), D1-PR (FAM-CAT GTG GTT GGG AGC ACG C-BHQ1) for DEN-1; D2-F (CAG GTT ATG GCA CTG TCA CGA T), D2-R (CCA TCT GCA GCA ACA CCA TCT C), D2-PR (HEX-CTC TCC GAG AAC AGG CCT CGA CTT CAA-BHQ1) for DENV-2; D3-F (GGA CTGG ACA CAC GCA CTC A), D3-R (CAT GTC TCT ACC TTC TCG ACT TGT CT), D3-PR (TexasRED-ACC TGG ATG TCG GCT GAA GGA GCT TG-BHQ2) for DENV-3 and; D4-F (TTG TCC TAA TGA TGC TGG TCG), D4-R (TCC ACC TGA GAC TCC TTC CA), D4-PR (CY5-TTC CTA CTC CTA CGC ATC GCA TTC CG-BHQ3) for DENV-4. All the primers and probes were ordered from Proligo (Sigma, Singapore). After this step, the PCR tubes were incubated in the Rotor Gen Q instruments (Qiagen, Malaysia) and ran with thermal cycles at $45^{\circ} \mathrm{C}$ for $10 \mathrm{~min}$ to reverse transcriptase (RT), at $95^{\circ} \mathrm{C}$ for $10 \mathrm{~min}$ to destroy the RT enzyme, 40 cycles with two thermal steps at $95^{\circ} \mathrm{C}$ for $15 \mathrm{~s}$ followed by $60^{\circ} \mathrm{C}$ for one minute in which we recorded fluorescent signals. The results were read and characterized together with DENV serotypes based on amplifying signals dyed with four different color channels: FAM for type 1, HEX for type 2, TexasRED for type 3, and CY5 for type 4. If signals were absent in all channels, we ran another assay using RT-rPCR mix manufactured by Nam Khoa company for house-keeping gene, RNAseP, to confirm the negativity of the sample

The enrolled patients were also provided a dengue NS1 rapid test by the laboratory of the Quang Nam Region General Hospital. This test was based on the immunochromatography lateral flow that could detect the NS1 antigen of DENV circulated in the blood of the patients during the infection, as per manufacturer description (SD BIOLINE DENGUE NS1 AG, SD manufacturer).

For statistical analyses, the SPSS 17.0 statistical software was used as a statistical procedure for the analysis of these data. The Chi-square procedure was used for all counting materials and a t-test for measurement material. Laboratory data were then compared between the groups using single-factor ANOVA analysis $(p<0.05$ was considered statistically significant).

\section{Results}

From December 2018 to February 2019, 488 patients were enrolled in the study, including 111 children and 377 adults. Among these patients, 336, including 74 children (22.02\%) and 262 adults $(77.98 \%)$, were diagnosed with DHF based on the positive results either from RT-rPCR or NS1 tests. The proportion of males and females in 74 pediatric patients were $59.46 \%$ (44/74) and 40.54\% (30/74), respectively; among the 262 adults, $48.47 \%(127 / 262)$ were men and 51.53\% (135/262) were women. The RT-rPCR detected DENV in 273 patients with the following different percentages of DENV serotypes: 12.82\% (35/273) DENV-1, 17.95\% (49/273) DENV-2, 0.37\% (1/273) DENV-3, and 68.50\% (187/273) DENV-4. There was one case $(0.37 \%)$ of co-infection of DEN-2 with DENV-4. The percentages of DENV serotypes in our study are shown on the Figure 1. 


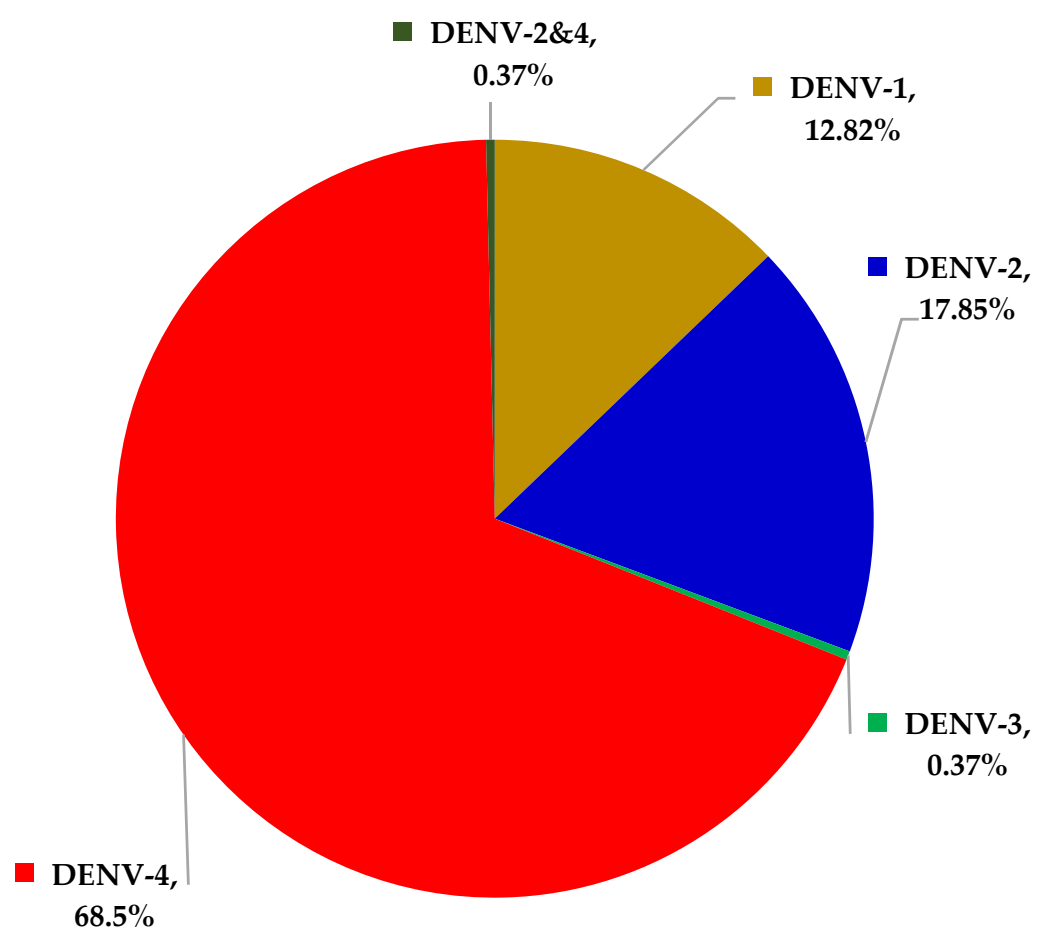

Figure 1. Percentages of DENV serotypes among 273 patients who tested positive for DENV by RT-rPCR.

The analysis of the RT-rPCR and the NS1 results showed that among 336 confirmed patients affected by DHF, 273 tested positive by RT-rPCR, and 304 tested positive with NS1. The results of both the RT-rPCR and the NS1 tests on 488 participants are resumed in Table 1.

Table 1. Results of qPCR assay and NS1 tests in the overall 488 participants.

\begin{tabular}{cccccc}
\hline & & \multicolumn{2}{c}{ One-Step RT-rPCR } & \multirow{2}{*}{ Total } \\
\hline \multirow{3}{*}{ NS1 } & & {$[+]$} & {$[-]$} & 63 & 304 \\
& & {$[+]$} & 241 & 152 & 184 \\
\cline { 3 - 6 } & & {$[-]$} & 273 & 215 & 488 \\
\hline
\end{tabular}

The sensitivity in the first 3 days of the diseases of the RT-rPCR assay and NS1 test in the diagnosis of DHF described in this study (Table 2) showed around 92.3\% (108/117) and $89.7 \%(105 / 117)$ values, respectively. However, these differences were not statistically significant $(t=0.686, p>0.05)$. From day 4 to 9 of the disease, the sensitivity of RT-rPCR gradually decreased from $82 \%$ to $33 \%$ on day 8 ; the test was not able to detect DENV on day 9. The average sensitivity of the test during this time was $75 \%(141 / 188)$. On the contrary, the average sensitivity of the NS1 test was continuously high $(89.9 \%=169 / 188)$ from day 4 and even on day 9 .

The difference between the sensitivity of the RT-rPCR assay and the NS1 rapid test was statistically significant $(t=3.796, p<0.05)$. Table 2 shows the sensitivity of the RT-rPCR and the NS1 in the diagnosis of DHF per day of infection. The sensitivity of the test per day of infection was defined by the percentage of the DHF cases detected by the test on the overall DHF cases. For example, among 85 cases of DHF with D4 infection, 70 were detected by RT-rPCR and 76 were detected by NS1; then, the sensitivity of RT-rPCR was $82.35 \%(70 / 85)$, and the sensitivity of NS1 was $89.41 \%(76 / 85)$. 
Table 2. The sensitivity of RT-rPCR and NS1 in diagnosis of DHF per day of infection.

\begin{tabular}{cccccc}
\hline Day of Infection & DHF Case & NS1(+) Case & RT-rPCR(+) Case & Sensitivity of NS1 & $\begin{array}{c}\text { Sensitivity of } \\
\text { RT-rPCR }\end{array}$ \\
\hline D9 & 1 & 1 & 0 & $100.00 \%(1 / 1)$ & $0.00 \%(0 / 1)$ \\
\hline D8 & 3 & 3 & 1 & $100.00 \%(3 / 3)$ & $33.33 \%(1 / 3)$ \\
\hline D7 & 11 & 10 & 7 & $90.91 \%(10 / 11)$ & $63.64 \%(7 / 11)$ \\
\hline D6 & 28 & 24 & 16 & $85.71 \%(24 / 28)$ & $57.14 \%(16 / 28)$ \\
\hline D5 & 60 & 55 & 47 & $91.67 \%(55 / 60)$ & $78.33 \%(47 / 60)$ \\
\hline D4 & 85 & 76 & 70 & $89.41 \%(76 / 85)$ & $82.35 \%(70 / 85)$ \\
\hline D3 & 75 & 68 & 33 & $90.67 \%(68 / 75)$ & $94.67 \%(71 / 75)$ \\
\hline D2 & 38 & 34 & 4 & $75.47 \%(34 / 38)$ & $86.84 \%(33 / 38)$ \\
\hline D1 & 4 & 3 & 0 & & $100.00 \%(4 / 4)$ \\
\hline D0 & 0 & 0 & 24 & $96.77 \%(30 / 31)$ & $77.42 \%(24 / 31)$ \\
\hline Undetermined & 31 & 30 & 273 & $90.48 \%(304 / 336)$ & $81.25 \%(273 / 336)$ \\
\hline Total & 336 & 304 & & &
\end{tabular}

As shown in Table 3, most clinical manifestations in the 336 DHF patients were fever, headache, orbital pain, and joint pain, which occurred in $80-90 \%$ of the patients. Among these manifestations, orbital pain and joint pain appeared more frequently in adults than children. The explanation for this difference may be due to children's behavior that usually tends not to show this type of malaise. Lethargy and increased liver enzymes were the second-highest symptom manifestation in about $40-45 \%$ of the patients. Hematological findings such as Hematocrit (Hct) $>42 \%$, thrombocytopenia, and cutaneous hemorrhage were present in about $22-33 \%$ of the patients. Gastrointestinal (GI) bleeding, hematuria, fluid accumulation detected by ultrasounds, and thrombocytopenia $<20,000$ were rare events. More than half of the patients $(56.25 \%)$ were classified as DHF with warning signs, manifesting at least one of these signs: lethargy, mucous membrane hemorrhages, liver enzyme increase, Hct increase, and rapid platelet decrease. However, there was no one classified as severe DHF, probably because severe DHF patients were referred to higher-level hospitals (often located in the big cities outside the rural area under investigation) before the study was conducted. Apart from subjective manifestations, there was no other difference in clinical settings between children and adults diagnosed with DHF in our study.

Table 3. Proportion of clinical manifestations in 336 patients diagnosed with DHF.

\begin{tabular}{|cccc}
\hline & Children & Adults & Total \\
\hline Fever & 93.15 & 92.09 & 92.33 \\
Orbital pain & 79.71 & 90.28 & 87.97 \\
Muscular/joint pain & 68.12 & 86.59 & 82.54 \\
Positive tourniquet test & 0 & 0.82 & 0.63 \\
Cutaneous hemorrhages & 25.00 & 21.63 & 22.36 \\
Gum bleeding & 13.24 & 15.10 & 14.70 \\
Mucous membrane hemorrhages & 2.94 & 2.04 & 2.24 \\
Lethargy & 35.29 & 47.76 & 45.05 \\
Abdominal pain & 2.70 & 0.78 & 1.21 \\
Hepatomegaly & 0 & 0 & 0 \\
Hct increase $>42 \%$ & 24.66 & 28.19 & 27.41 \\
Platelet decrease $<20 \mathrm{~K}$ & 2.70 & 7.72 & 6.61 \\
Platelet decrease $<100 \mathrm{~K}$ & 25.68 & 35.91 & 33.63 \\
DHFD with warning signs & 51.35 & 57.63 & 56.25 \\
Liver enzyme increase & 33.78 & 41.31 & 39.64 \\
Abdominal fluid accumulation & 0 & 2.32 & 1.80 \\
\hline
\end{tabular}


Table 4 shows clinical manifestations and subclinical findings associated with different DENV serotypes in patients diagnosed with DHF. The table only shows DENV-1, -2 , and -4 because DENV-3 was detected in a few cases, and DENV-2 and -4 co-infection was detected merely in one patient. As the results show in Table 4, in our study, there were almost similar clinical manifestations and subclinical findings various among DENV serotypes. These results could be explained by the absence of severe DHF cases; thereby, we were not able to analyze the difference in clinical manifestations and subclinical findings among DENV serotypes in our DHF patients. The outcomes also revealed a small number of gastrointestinal bleedings $(2.22 \%)$, severe thrombocytopenia $<10,000$, and abdominal fluid retention $(8.33 \%)$, features that were observed only in patients infected with DENV-2. These manifestations were very rare events in patients diagnosed with different DENV serotypes.

Table 4. Clinical manifestations and subclinical findings associated with different DENV serotypes in patients diagnosed with DHF.

\begin{tabular}{|cccc}
\hline & DENV-1 & DENV-2 & DENV-4 \\
\hline Sustained fever & 63.64 & 72.34 & 69.73 \\
Fever & 83.33 & 92.31 & 78.57 \\
Orbital pain & 78.13 & 88.89 & 91.67 \\
Muscular/joint pain & 78.13 & 88.89 & 81.01 \\
Positive tourniquet test & 0 & 0 & 1.12 \\
Cutaneous hemorrhages & 31.25 & 11.11 & 17.42 \\
\hline Gum bleeding & 15.63 & 11.11 & 14.04 \\
Subconjunctival hemorrhage & 0 & 0 & 0.56 \\
Hematuria & 3.13 & 0 & 0 \\
GI bleeding & 0 & 2.22 & 0 \\
Lethargy & 46.88 & 37.78 & 47.75 \\
Abdominal pain & 0 & 2.08 & 1.62 \\
Hepatomegaly & 0 & 0 & 0 \\
Hct $\geq 42 \%$ & 2.94 & 12.5 & 9.14 \\
PLT <10,000 & 0 & 4.17 & 0 \\
PLT 10,000-20,000 & 0.03 & 0.06 & 0.06 \\
PLT 20,000-100,000 & 44.12 & 39.58 & 29.41 \\
\hline DHFD with warning signs & 48.57 & 59.18 & 59.36 \\
\hline Liver enzymes $>40-<400$ & 50 & 37.5 & 36.02 \\
Abdominal fluid (ultrasounds) & 0 & 8.33 & 1.61 \\
\hline
\end{tabular}

\section{Discussion}

While Asian countries often experience DHF outbreaks, which rarely affect children under 15 of age, in South America, DHF widely spreads among the population independently from the age, probably due to different lifestyles [18-21]. In this study, 77.98\% of infected patients were adults, more than three folds compared to children $(22.02 \%)$, with data statistically significant $(\mathrm{t}=14.505, p<0.001)$.

An individual primarily infected with DENV could create specific antibodies for the primary serotype; however, these antibodies not only could not be enough to fight against other serotypes but also trigger clinical manifestations when patients are secondarily infected with other DENV serotypes. It was probably because these useless antibodies increased the adhesive and invasive ability of DENV into growing cells, promoting the immune reaction through releasing inflammatory cytokines such as IL6, IL1, TNF, and IFN. The main consequence of this uncontrolled inflammatory condition is the increased permeability of vessels and blood coagulation, as proposed by the theory of antibodydependent enhancement [22] in the physiopathology of DHF. Although the theory has not demonstrated this so far, it was the only explanation for the physiopathology of DHF and the ability to cause the outbreak of the DHF when the distribution of DENV serotypes change in various regions. Cummings et al. reported DENV serotypes changes in cycles of 8-10 years in Thailand [23]. From 1980 to 2000, Adam et al. also showed that DHF epidemics were caused by a rotation of three DENV serotypes: DENV-1, DENV-2, and 
DENV-3 [24]. The assumption could be based on two reasons. First, infected individuals may generate antibodies explicitly against only one serotype while remaining vulnerable to others, making new outbreaks always an open possibility. Second, the different serotypes are always available in mosquitos; thus, they not only endemically maintain the disease but also ready make the outbreak with new serotypes.

The outcomes of DENV serotype analysis in Vietnam, obtained during the largest DHF outbreak in 1987, showed 83,905 infections and 904 deaths mainly caused by DENV-2 (90.5\%). On the other hand, in 1990, DENV-1 appeared and soon spread widely, reaching $62.5 \%$ contagious during 1993. DENV-3 was also detected with a lower proportion during the same period. From 1994 to 1998, scientists isolated the DENV-3, which showed the following decrease of DENV-1 distribution. In 1998, the prominent DENV serotype isolated was again the DENV-3 [25]. Across the years, the DENV-3 steadily decreased until it was completely replaced by other serotypes in 2000. Our findings showed that DENV-4 (68.5\%) was prominent in the recent DHF outbreak in Quang Nam province (2018), followed by DENV-2 (17.9\%), DENV-1 (12.8\%), and DENV-3 (1 case, $0.4 \%)$.

When comparing data from studies conducted in HCM city in four consecutive years, from 2010 to 2013 [26], we could find the prominent distribution of DENV-1 (36\%), followed by DENV-4 (27\%), DENV-2 (26\%), and DENV-3 (11\%). While a different study conducted in Dong Thap province during the same period [27] from February 2012 to February 2013, the outcomes showed a different scenario: the DENV-2 distribution was the highest (36\%), followed by DENV-4 (35\%), DENV-3 (19\%), and DENV-1 (11\%). Based on the data provided above, we could recognize that the prominent distribution of DENV serotypes was likely to differ between regions but similar in trend of the increasingly prominent distribution of DENV-4 and the gradually decreasing of DENV-3. Therefore, longitudinal studies for the distribution of DENV serotypes are necessary for predicting the DHF outbreaks in a certain region and country. These studies will also be the trend of our DHF research in the future.

In addition, several studies revealed the relationship between DENV serotypes and the severity of DHF [10-13], showing a strict bond existing between the virus, host, and the environment. By this mechanism, the severity of DHF outbreak could be predicted based on the identification of DENV serotype characterized in the previous outbreak [11,12]. This aspect could be the main topic of investigation in future studies as a starting point for the creation of a specific algorithm and for the development of a more precise diagnostic approach based on targeting antibodies for each of the DENV serotypes.

At the time of this article, the antibody (IgG or $\operatorname{IgM})$ assays represented valuable tools in diagnosis whether patients were facing a primary or secondary infection. NS1 tests were highly valuable as rapid tests based on immune chromatography, which is an easily assessed procedure in laboratories or even on-site in the clinical wards [28]. There are many manufacturers that can provide the basic kits needed to perform these tests that can reach a specificity as higher as $90 \%$ with a grade of high sensitivity that can be stable for the first three days [28]. The NS1 tests performed in Region General Hospital of Quang Nam province were highly sensitive, like the RT-rPCR assay in the first three days, and were stable until day 9 of the condition. However, due to the limitation of blood samples on day 8 and day 9 , it was difficult to consolidate these results.

\section{Conclusions}

Although DHF outbreaks are worsening in Quang Nam province with the increasing numbers of infections, studies describing the distribution of DENV serotypes are rare. Assessing the circulation and the grade of transmission of DENV serotypes could help local health authorities in predicting new foci of infection, thus allowing them to implement proper treatment strategies at a local level and nationwide. Owing to the application of RT-rPCR assay in detecting and serotyping DENV in patients' blood samples, our study could be helpful in elucidating the question regarding which of the DENV serotype would be prominent during the next DHF outbreak. We plan to continue the study in the next years to better describe the DHF epidemiology in Vietnam. 
Author Contributions: Conceptualization, V.H.P., D.Q.P., L.D.N.N. and C.G.I.; methodology, V.H.P., K.C.D.N., D.K.T. and D.T.P.; editing, G.D., F.I., S.K.A., A.M. and P.P.; validation, V.H.P., C.G.I. and A.B.; formal analysis, V.H.P., H.T.P., S.T.P., P.P., F.I., S.S. and A.M.; investigation, S.H.L. and A.B.; bibliographic research, T.N., P.T.T.P., S.T.H.N., T.T.P., A.D.I., S.C. and P.P. All authors have read and agreed to the published version of the manuscript.

Funding: This research received no external funding.

Institutional Review Board Statement: Patients enrolled in the study were all subjects clinically diagnosed with dengue hemorrhagic fever (DHF) and hospitalized at Quang Nam General Hospital, in accordance with decision number 1499 issued on 17 May 2011, by the Ministry of Health.

Informed Consent Statement: Informed consent was obtained from all subjects involved in the study. Written informed consent was obtained from the patient(s) to publish this paper.

Data Availability Statement: Data can be accessed upon request to van.pham@pctu.edu.vn (V.H.P.).

Acknowledgments: We would like to express our deep gratitude to doctors and medical staff in the infectious disease department of Quang Nam Region General hospital for their corporation. We would also like to thank Nam Khoa company and the International Institute of Gene and Immunology for providing the RT-rPCR kits and protocols; our grateful thanks are also extended to Phan Chau Trinh University of Medicine for providing us facilities to conduct the study.

Conflicts of Interest: The authors declare no conflict of interest.

Ethical Approval: Local institutional review board approved this study.

\section{References}

1. Katzelnick, L.C.; Coloma, J.; Harris, E. DHFD: Knowledge gaps, unmet needs, and research priorities. Lancet Infect. Dis. 2017, 17, e88-e100. [CrossRef]

2. Hermann, L.L.; Gupta, S.B.; Manoff, S.B.; Kalayanarooj, S.; Gibbons, R.V.; Coller, B.A. Advances in the understanding, management, and prevention of DHFD. J. Clin. Virol. 2015, 64, 153-159. [CrossRef] [PubMed]

3. Nujum, Z.T.; Thomas, A.; Vijayakumar, K.; Nair, R.R.; Pillai, M.R.; Indu, P.S.; Sundar, S.; Gopakumar, S.; Mohan, D.; Sudheeshkumar, T.K. Comparative performance of the probable case definitions of DHFD by WHO (2009) and the WHO-SEAR expert group (2011). Pathog. Glob. Health 2014, 108, 103-110. [CrossRef] [PubMed]

4. Buonora, S.N.; Passos, S.R.L.; Daumas, R.P.; Machado, M.G.L.; Berardinelli, G.M.; de Oliveira, D.N.R.; de Oliveira, R.V.C. Pitfalls in acute febrile illness diagnosis: Interobserver agreement of signs and symptoms during a DHFD epidemics. J. Clin. Nurs. 2020, 29, 1590-1598. [CrossRef] [PubMed]

5. Aubry, M.; Mapotoeke, M.; Teissier, A.; Paoaafaite, T.; Dumas-Chastang, E.; Giard, M.; Cao-Lormeau, V.M. DHFD virus serotype 2 (DENV-2) epidemics, French Polynesia 2019. Euro Surveill. 2019, 24, 1900407. [CrossRef] [PubMed]

6. Rajapakse, S.; de Silva, N.L.; Weeratunga, P.; Rodrigo, C.; Fernando, S.D. Prophylactic and therapeutic interventions for bleeding in DHFD: A systematic review. Trans. R. Soc. Trop. Med. Hyg. 2017, 111, 433-439. [CrossRef]

7. López-Díaz, T.; Lugo, F.; Rodríguez, J.M.; Sabao, E.; Sierra, K.; Valdés, Y.; Vera, J. Compliance with management guidelines in patients with suspected DHFD. Bol. Asoc. Med. Puerto Rico 2016, 108, 53-56.

8. Laodong. Available online: https:laodong.vn/y-te/dich-sot-xuat-huyet-tang-cao-tai-quang-nam-747103.ldo (accessed on 10 March 2019).

9. Guzman, M.G.; Alvarez, M.; Halstead, S.B. Secondary infection as a risk factor for DHFD hemorrhagic fever/DHFD shock syndrome: An historical perspective and role of antibody-dependent enhancement of infection. Arch. Virol. 2013, 158, 1445-1459. [CrossRef]

10. Fried, J.R.; Gibbons, R.V.; Kalayanarooj, S.; Thomas, S.J.; Srikiatkhachorn, A.; Yoon, I.K.; Jarman, R.G.; Green, S.; Rothman, A.L.; Cummings, D.A. Serotype-Specific Differences in the Risk of DHFD Hemorrhagic Fever: An Analysis of Data Collected in Bangkok, Thailand from 1994 to 2006. PLoS Negl. Trop. Dis. 2010, 4, e617. [CrossRef]

11. Alvarez, M.; Rodriguez-Roche, R.; Bernardo, L.; Vázquez, S.; Morier, L.; Gonzalez, D.; Castro, O.; Kouri, G.; Halstead, S.B.; Guzman, M.G. DHFD Hemorrhagic Fever Caused by Sequential DHFD 1-3 Virus Infections Over a Long Time Interval: Havana Epidemic, 2001-2002. Am. J. Trop. Med. Hyg. 2006, 75, 1113-1117. [CrossRef]

12. Aguas, R.; Dorigatti, I.; Coudeville, L.; Luxemburger, C.; Ferguson, N.M. Cross-serotype interactions and disease outcome prediction of DHFD infections in Vietnam. Sci. Rep. 2019, 9, 9395. [CrossRef] [PubMed]

13. Halstead, S.B.; Lan, N.T.; Myint, T.T.; Shwe, T.N.; Nisalak, A.; Kalyanarooj, S.; Nimmannitya, S.; Soegijanto, S.; Vaughn, D.W.; Endy, T.P. DHFD hemorrhagic fever in infants: Research opportunities ignored. Emerg. Infect. Dis. 2002, 8, 1474-1479. [CrossRef] [PubMed] 
14. Santiago, G.A.; Vergne, E.; Quiles, Y.; Cosme, J.; Vazquez, J.; Medina, J.F.; Medina, F.; Colón, C.; Margolis, H.; Muñoz-Jordán, J.L. Analytical and clinical performance of the CDC real time RT-PCR assay for detection and typing of DHFD virus. PLoS Negl. Trop. Dis. 2013, 7, e2311. [CrossRef]

15. Santiago, G.A.; Vázquez, J.; Courtney, S.; Matías, K.Y.; Andersen, L.E.; Colón, C.; Butler, A.E.; Roulo, R.; Bowzard, J.; Villanueva, J.M.; et al. Performance of the Trioplexreal-time RT-PCR assay for detection of Zika, DHFD, and chikungunya virus. Nat. Commun. 2018, 9, 1391. [CrossRef]

16. Pham, V.H. The solution for the low-income countries to establish the automatic extraction of the nucleic acid from the clinical samples. In Proceedings of the Asean Congress on Medical Biotechnology and Molecular Biosciences, Bangkok, Thailand, 8-9 October 2015.

17. Pham, V.H.; Thanh, B.T.; Quoc, N.V. Production and Evaluation of the Kit Using Magnetic Silica Coated Nano-Ironbeads to Extract the Nucleic Acid from Different Samples; ISAAR, BEXCO: Busan, Korea, 2017.

18. Gubler, D.J. Epidemic DHFD/DHFD hemorrhagic fever as a public health, social and economic problem in the 21st century. Trends. Microbiol. 2002, 10, 100-103. [CrossRef]

19. Stephan, C.; Allwinn, R.; Brodt, H.R.; Knupp, B.; Preiser, W.; Just-Nübling, G. Travel-acquired DHFD infection: Clinical spectrum and diagnostic aspects. Infections 2002, 30, 225-228. [CrossRef]

20. Patumanond, J.; Tawichasri, C.; Nopparat, S. DHFD hemorrhagic fever, Uttaradit, Thailand. Emerg. Infect. Dis. 2003, 9, 1348-1350. [CrossRef]

21. Simmons, M.; Burgess, T.; Lynch, J.; Putnak, R. Protection against DHFD virus by non-replicating and live attenuated vaccines used together in a prime boost vaccination strategy. Virology 2010, 396, 280-288. [CrossRef]

22. Tsai, T.T.; Chuang, Y.J.; Lin, Y.S.; Wan, S.W.; Chen, C.L.; Lin, C.F. An emerging role for the anti-inflammatory cytokine interleukin10 in DHFD virus infection. J. Biomed. Sci. 2013, 20, 40. [CrossRef]

23. Cummings, D.A.; Irizarry, R.A.; Huang, N.E.; Endy, T.P.; Nisalak, A.; Ungchusak, K.; Burke, D.S. Travelling waves in the occurrence of DHFD haemorrhagic fever in Thailand. Nature 2004, 427, 344-347. [CrossRef]

24. Adams, B.; Holmes, E.C.; Zhang, C.; Mammen, M.P., Jr.; Nimmannitya, S.; Kalayanarooj, S.; Boots, M. Cross-protective immunity.can account for the alternating epidemic pattern of DHFD virus.serotypes circulating in Bangkok. Proc. Natl. Acad. Sci. USA 2006, 103, 14234-14239. [CrossRef] [PubMed]

25. Phuong, C.X.; Nhan, N.T.; Kneen, R.; Thuy, P.T.; van Thien, C.; Nga, N.T.; Thuy, T.T.; Solomon, T.; Stepniewska, K.; Wills, B.; et al. Clinical diagnosis and assessment of severity of confirmed DHFD infection in Vietnamese childrens: Is the WHO classification system helpful? Am. J. Trop. Med. Hyg. 2004, 70, 172-179. [CrossRef] [PubMed]

26. Quoc, C.H.; Henrik, S.; Isabel, R.B.; In-Kyu, Y.; Chau, N.V.; Hung, N.T.; Tuan, H.M.; Lan, P.T.; Willis, B.; Nisalak, A.; et al. Synchrony of DHFD Incidence in Ho Chi Minh City and Bangkok. PLoS Negl. Trop. Dis. 2016, 10, e0005188. [CrossRef]

27. Lien, P.T.K.; Briant, L.; Tang, T.B.; Trang, B.M.; Gavotte, L.; Cornillot, E.; Duoc, V.T.; Duong, T.N.; Frutos, R.; Nga, P.T. Surveillance of DHFD and chikungunya infection in Dong Thap, Vietnam: A 13-month study. Asian Pac. J. Trop. Med. 2016, 9, 39-43. [CrossRef]

28. Blacksell, S.D. Commercial DHFD Rapid Diagnostic Tests for Point-of-Care Application: Recent Evaluations and Future Needs? J. Biomed. Biotechnol. 2012, 2012, 151967. [CrossRef] [PubMed] 